\title{
Multi-subtype Natural Human Leukocyte Interferon Alpha
}

National Cancer Institute

\section{Source}

National Cancer Institute. Multi-subtype Natural Human Leukocyte Interferon Alpha. NCI

Thesaurus. Code C97509.

A preparation containing a mixture of multiple naturally occurring, active subtypes 1, 2, 8, 10, 14 and 21 of interferon alpha (IFN-alpha) with immunomodulating, anti-viral and anticancer activities. Multi-subtype natural human leukocyte IFN-alpha is purified from the leukocyte fraction of human blood challenged with Sendai virus. Upon administration, IFN-alpha subtypes bind to cell surface IFN-alpha receptors (IFNARs), resulting in an upregulation of interferon stimulated genes and related protein products. This ultimately leads to the proliferation of human B cells, activation of natural killer (NK) cells and dendritic cells (DCS), an increase in HLA-I and HLA-II expression and activation of CD8lymphocytes. Compared to sing le-subtype IFN, multi-subtypes act synergistically. 